\title{
APORTE E ADEQUAÇÃO NUTRICIONAL ANTES E DURANTE A GESTAÇÃO: ESTU- DO DE ACOMPANHAMENTO NA ILHA DO FAIAL
}

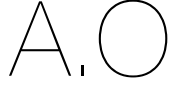

ARTIGO ORIGINAL

1 Unidade de Saúde da llha do Faial, Vista Alegre, 9901-853 Horta, Açores, Portugal

2 Faculdade de Ciências da Nutrição e Alimentação da Universidade do Porto, Rua do Campo Alegre, n. ${ }^{\circ} 823$,

4150-180 Porto, Portugal

${ }^{3}$ EPIUnit - Instituto de Saúde Pública da Universidade do Porto, Rua das Taipas, n. ${ }^{\circ} 135$ 4050-600 Porto, Portugal

*Endereço para correspondência:

Frederico Viveiros

ua Alameda Barão de Roches, n. ${ }^{3} 33-\mathrm{A}$,

9900-104 Horta, Açores,

Portugal

fredericocviveiros@gmail.com

Histórico do artigo:

Recebido a 2 de maio de 2021 Aceite a 16 de novembro de 2021

\section{NUTRITIONAL INTAKE AND ADEQUACY BEFORE AND DURING GESTATION: A LONGITUDINAL STUDY IN THE FAIAL ISLAND}

\section{RESUMO}

A nutrição afeta a saúde materna e das futuras gerações. São vários os estudos que reportam um inadequado aporte nutricional antes da conceção e durante a gestação.

Pretendeu-se avaliar o aporte nutricional de mulheres da ilha do Faial (Açores) antes e durante a gravidez e analisar a sua adequação. Neste estudo prospetivo foram analisados dados de 34 mulheres.

Para avaliar a ingestão alimentar foi aplicado um questionário de frequência de consumo alimentar semi-quantitativo, em dois momentos: na primeira consulta pré-natal (primeiro trimestre de gestação) e na última consulta pré-natal (final do terceiro trimestre de gestação). Para analisar a adequação do aporte nutricional foram utilizadas, sempre que possível, as recomendações da European Food Safety Authority, 2017.

Os nutrientes com maior inadequação antes da conceção foram: vitamina D (100\%), iodo (76,5\%), lípidos (73,5\%), hidratos de carbono $(55,9 \%)$ e fibra $(44,1 \%)$. Na gestação, para além dos quatro nutrientes mencionados anteriormente, os folatos também apresentaram uma elevada prevalência de inadequação (58,8\%). Neste período, verificou-se que os folatos e o iodo foram os nutrientes com maior aumento na inadequação (44,1 e 20,6 pontos percentuais, respetivamente).

Os resultados deste estudo reforçam a importância da adesão à suplementação, avaliação da adequação do aporte nutricional e da individualização da terapêutica nutricional.

\section{PALAVRAS-CHAVE}

Adequação nutricional, Aporte nutricional, Gestação, Pré-conceção

ABSTRACT

Nutrition affects both maternal and future generations' health. There are several studies reporting an inadequate nutritional supply before conception and during pregnancy.

It was intended to assess the nutritional intake of women from the island of Faial (Azores) before and during pregnancy and to analyze its adequacy. In this prospective study, data from 34 women were analyzed.

To assess food intake, a semi-quantitative food frequency questionnaire was applied in two moments: first pre-birth appointment (first trimester of gestation) and last pre-birth appointment (end of the third trimester of gestation). To analyze the adequacy of nutritional support, the recommendations of the European Food Safety Authority, 2017 were used, whenever possible.

The nutrients with higher inadequacy before conception were: vitamin D (100\%), iodine (76.5\%), lipids (73.5\%), carbohydrates (55.9\%) and fiber (44.1\%). During pregnancy, in addition to the four mentioned above, folates also had a high prevalence of inadequacy (58.8\%). In this period, we found that folates and iodine were the nutrients with the greatest increase in inadequacy (44.1 and 20.6 percentage points, respectively).

The results of this study reinforce the importance of promoting the adherence to supplementation, the assessment of the adequacy of nutritional intake and the individualization of nutrition therapy.

KEYWORDS

Nutritional adequacy, Nutritional intake, Gestation, Preconception

\section{INTRODUÇÃO}

A nutrição é fundamental em todas as fases da vida, particularmente durante a gestação pois não só afeta a saúde materna como também influencia a saúde das futuras gerações (1-3). Este efeito é particularmente importante nos primeiros mil e cem dias (desde a pré-conceção até aos vinte e quatro meses de vida) visto que desempenha um papel fundamental na modulação metabólica do feto e do lactente, com potenciais resultados a longo prazo para a saúde do futuro adulto $(4,5)$

A investigação reporta prevalências elevadas de défices nutricionais antes da conceção (6-8). Atualmente existe evidência científica que comprova a associação entre um inadequado estado nutricional pré-concecional e problemas 
perinatais (9-11). São vários os estudos que reportam um inadequado aporte nutricional durante a gestação $(6-8,12-21)$. A inadequação nutricional tem consequências negativas quer para a mãe quer para a criança, uma vez que a malnutrição materna pode estar associada a efeitos adversos na gravidez e no pós-parto (22-25).

Para além da maior motivação para a mudança comportamenta por parte deste público-alvo, o facto de este estudo ser o único que utiliza os valores de referência da European Food Safety Authority (EFSA), especificamente desenvolvidos para a população europeia para avaliar a adequação nutricional e também por ser o primeiro estudo sobre esta temática, realizado na Região Autónoma dos Açores e apenas o segundo feito a nível nacional, reforça a sua pertinência.

\section{OBJETIVOS}

Avaliar o aporte nutricional de mulheres da ilha do Faial, antes e durante a gravidez e analisar a sua respetiva adequação.

\section{METODOLOGIA}

Este estudo foi aprovado pela Comissão de Ética para a Saúde da Unidade de Saúde da llha do Faial, pelo parecer número 4.2018, a 3 de maio de 2018. Todas as participantes deram o seu consentimento informado por escrito e foi-lhes atribuído individualmente um código para assegurar a sua confidencialidade. Foi igualmente assegurada a anonimização dos dados recolhidos.

A amostra deste estudo foi composta por grávidas que completaram a sua gestação entre maio de 2018 e junho de 2019 e teve como critérios de inclusão mulheres com idade igual ou superior a dezoito anos, idade gestacional inferior a doze semanas, sem comorbilidades, residentes na ilha do Faial, inscritas na Unidade de Saúde desta ilha e sem qualquer situação que impossibilitasse a participação livre e informada. As mulheres com história de abortos espontâneos, morte fetal, partos prematuros (inferior a 32 semanas de gestação) e/ou previamente submetidas a cirurgia bariátrica foram excluídas.

Um total de 48 grávidas foram convidadas a participar no estudo, seis $(12,5 \%)$ das quais recusaram. Foram posteriormente excluídos os dados de oito participantes: três sofreram aborto espontâneo, uma teve parto prematuro (inferior a 32 semanas de gestação), duas tiveram diagnóstico de diabetes gestacional e duas desistiram do estudo. Desta forma, foram analisados os dados de 34 mulheres.

Para a colheita de dados sobre a ingestão alimentar foi aplicado o questionário de frequência alimentar (QFA), versão semi-quantitativa (26), que se encontra validada para a população portuguesa (27) e, em concreto, para as gestantes (28). Este questionário foi aplicado em dois momentos, o primeiro dos quais na primeira consulta pré-natal (primeiro trimestre de gestação), com a finalidade de estimar o aporte nutricional usual antes da conceção. O segundo foi aplicado na última consulta pré-natal (final do terceiro trimestre de gestação), para estimar o aporte nutricional usual durante a gravidez. A conversão dos alimentos em nutrientes foi efetuada utilizando o programa informático Food Processor Plus ${ }^{\circledR}$ (ESHA Research, Salem, Oregon), com informação nutricional proveniente de tabelas de composição de alimentos do Departamento de Agricultura dos Estados Unidos da América, adaptada a alimentos tipicamente portugueses (26).

Para a avaliação da adequação do aporte nutricional foram utilizadas, sempre que possível, as recomendações da European Food Safety Authority (EFSA) uma vez que foram desenvolvidas para a população europeia (29). A média das necessidades energéticas, durante a gravidez, foi calculada adicionando $277 \mathrm{kcal}$ às necessidades anteriores à conceção. Este valor corresponde à média do aumento das necessidades energéticas nos três trimestres de gestação, cuja adequação teve como referência as average requirements (AR) (29). Para avaliar o exercício físico foi questionado se realizava algum tipo atividade física durante a gestação, o tipo de exercício praticado, a sua frequência e a respetiva duração. O nível de atividade física foi considerado como pouco ativo, à semelhança do evidenciado no inquérito alimentar nacional e de atividade física (IAN-AF) (30).

Para avaliar a adequação do aporte proteico foram utilizados os acceptable macronutrient distribution ranges (AMDR) (31). Relativamente à avaliação do aporte lipídico foram utilizadas as reference intake ranges for macronutrients (RI) (29). Na avaliação da adequação do aporte dos hidratos de carbono foi utilizado o AMDR porque, na referência anterior, não existem recomendações específicas deste macronutriente para este grupo (31). Pelo mesmo motivo, foi utilizado o valor da adequate intake (Al) para as fibras (31).

Para a avaliação da adequação do aporte de cálcio, ferro, vitamina A, tiamina, riboflavina, niacina e vitamina B6 foram utilizados os valores da AR (29). Para analisar os restantes micronutrientes, foram usados como pontos de corte as $\mathrm{Al}$, visto que nestes não existem valores de AR (32). Dada a impossibilidade de quantificar o consumo de fitatos, que podem influenciar negativamente a proporção de zinco disponível para absorção, foi utilizado o valor da estimated average requirement (EAR) para avaliar a adequação do aporte deste mineral (32). Para a avaliação da adequação da vitamina $\mathrm{C}$, também foi utilizado a EAR (32), porque nas recomendações europeias existe apenas a population reference intake para este nutriente (29).

A análise estatística foi realizada no programa IBM SPSS ${ }^{\circledR}$, versão 25.0 para Windows ${ }^{\circledR}$. Utilizou-se o teste de Kolmogorov-Smirnov para avaliar a normalidade das variáveis cardinais. A estatística descritiva consistiu no cálculo de frequências absolutas (n) e relativas (\%), médias e desvios-padrão (dp) e de medianas e percentis (P25; P75). Os testes t de Student e de Mann-Whitney foram usados para comparar, respetivamente, médias e ordens médias de amostras independentes, e o teste de Wilcoxon para comparar ordens médias de amostras emparelhadas. Utilizou-se o teste exato de Fisher para avaliar a independência entre pares de variáveis e o teste de McNemar para comparar proporções de amostras emparelhadas. Rejeitou-se a hipótese nula quando $p<0,05$.

\section{RESULTADOS}

Como é possível constatar na Tabela 1, a idade média das participantes foi de 31 anos $(\mathrm{dp}=4), 50 \%$ apresentava o ensino superior, $64,7 \%$ estava casada ou em união de facto, a maioria $(91,2 \%)$ estava empregada, mais de metade da amostra $(58,8 \%)$ era primípara e $79,4 \%$ considerou os seus rendimentos económicos suficientes para os seus gastos.

Na Tabela 2 apresentam-se os valores de referência, medianas do aporte energético e nutricional diário e prevalência de inadequação nutricional antes da conceção e durante a gestação. Os nutrientes com maior inadequação antes da conceção foram: vitamina D, iodo, lípidos, hidratos de carbono e fibra. Todos estes micronutrientes tiveram uma inadequação por defeito, à exceção dos lípidos que apresentaram uma inadequação por excesso. Na gestação, para além dos nutrientes mencionados anteriormente, os folatos também apresentaram uma elevada percentagem de inadequação por defeito. A inadequação por defeito de folatos e iodo acentuou-se na gestação, aumentando em 44,1 e 20,6 pontos percentuais, respetivamente, comparativamente à pré-conceção, sendo estes os nutrientes com maior aumento de inadequação.

Em termos de aporte energético, não se verificaram diferenças significativas entre os períodos pré-conceção e gestação. Considerando 
Tabela 1

Caracterização da amostra

\begin{tabular}{|c|c|c|c|}
\hline & n (\%) & & n (\%) \\
\hline \multicolumn{2}{|c|}{ Idade (anos) quando engravidou } & \multicolumn{2}{|l|}{ IMC pré-gestacional (kg/m²) } \\
\hline$\leq 29$ & $13(38,2)$ & {$[18,5 ; 25,0[$} & $17(50,0)$ \\
\hline 30 a 35 & $19(55,9)$ & {$[25,0 ; 30,0[$} & $9(26,5)$ \\
\hline$\geq 36$ & $2(5,9)$ & $\geq 30,0$ & $8(23,5)$ \\
\hline \multicolumn{2}{|l|}{ Escolaridade } & \multicolumn{2}{|l|}{ Número de filhos } \\
\hline 1. ${ }^{\circ}$ Ciclo incompleto & $2(5,9)$ & 0 & $20(58,8)$ \\
\hline 2. ${ }^{\circ}$ Ciclo incompleto & $3(8,8)$ & 1 & $12(35,3)$ \\
\hline 3. ${ }^{\circ}$ Ciclo incompleto & $1(2,9)$ & 2 & $1(2,9)$ \\
\hline 3. ${ }^{\circ}$ Ciclo completo & $3(8,8)$ & 3 & $1(2,9)$ \\
\hline Secundário incompleto & $3(8,8)$ & \multicolumn{2}{|l|}{ Patologias } \\
\hline Secundário completo & $5(14,7)$ & Síndrome vertiginoso & $1(2,9)$ \\
\hline Superior & $17(50,0)$ & Sinusite e psoríase & $1(2,9)$ \\
\hline \multicolumn{2}{|l|}{ Estado civil } & Rinite alérgica & $1(2,9)$ \\
\hline Solteira & $11(32,4)$ & Escoliose & $1(2,9)$ \\
\hline Casada ou em união de facto & $22(64,7)$ & Ovários poliquísticos & $1(2,9)$ \\
\hline Divorciada ou separada & $1(2,9)$ & \multicolumn{2}{|l|}{ Complicações reportadas } \\
\hline \multicolumn{2}{|l|}{ Situação profissional } & Náuseas e/ou vómitos & $17(50,0)$ \\
\hline Desempregada & $2(5,9)$ & Descolamento da placenta & $2(5,9)$ \\
\hline Empregada & $31(91,2)$ & Anemia & $3(8,8)$ \\
\hline Doméstica & $1(2,9)$ & Obstipação & $3(8,8)$ \\
\hline \multirow{2}{*}{\multicolumn{2}{|c|}{ IMC: Índice de Massa Corporal }} & Prática de atividade física & $21(61,8)$ \\
\hline & & Caminhada & $17(50,0)$ \\
\hline
\end{tabular}

Tabela 2

Valores de referência, mediana do aporte nutricional diário e prevalência de inadequação nutricional

\begin{tabular}{|c|c|c|c|c|c|c|c|c|c|}
\hline \multirow[b]{2}{*}{ NUTRIMENTOS } & \multicolumn{4}{|c|}{ PRÉ-CONCEÇÃO } & \multicolumn{4}{|c|}{ GESTAÇÃO } & \multirow[b]{2}{*}{$\mathrm{p}$} \\
\hline & VR & MEDIANA & P25; P75 & $\begin{array}{l}\text { INADEQUAÇÃO } \\
(\%)\end{array}$ & VR & MEDIANA & P25; P75 & $\begin{array}{c}\text { INADEQUAÇÃO } \\
(\%)\end{array}$ & \\
\hline Energia (kcal) & & 2599,9 & 2238,$1 ; 2961,9$ & & & 2765,0 & 2115,$5 ; 3130,4$ & & \\
\hline HC (\% VET) & $45-65^{a}$ & 44,2 & 41,$6 ; 50,0$ & 55,9 & $45-65^{a}$ & 44,6 & 40,$2 ; 49,6$ & 50,0 & 0,815 \\
\hline Lípidos (\% VET) & $20-35^{b}$ & 38,6 & 34,$6 ; 41,8$ & 73,5 & $20-35^{b}$ & 38,6 & 35,$3 ; 43,7$ & 82,4 & 0,549 \\
\hline AGS (\% VET) & & 10,1 & 8,$9 ; 11,9$ & & & 10,8 & 9,$4 ; 12,4$ & & \\
\hline AGMI (\% VET) & & 17,5 & 16,$5 ; 20,0$ & & & 18,5 & 16,$1 ; 21,9$ & & \\
\hline AGPI (\% VET) & & 6,1 & 5,$3 ; 7,0$ & & & 6,1 & 5,$6 ; 6,8$ & & \\
\hline Proteína (\% VET) & $10-35^{a}$ & 17,3 & 14,$4 ; 21,0$ & 0,0 & $10-35^{a}$ & 17,6 & 16,$4 ; 19,6$ & 0,0 & - - \\
\hline Vitamina A $(\mu \mathrm{g})$ & $490,0^{c}$ & 2653,1 & 623,$2 ; 4354,6$ & 0,0 & $540^{\circ}$ & 2558,8 & 1554,$7 ; 3790,6$ & 0,0 & --- \\
\hline Vitamina C (mg) & $60,0^{d}$ & 170,9 & 117,$2 ; 239,8$ & 5,9 & $70^{d}$ & 179,2 & 152,$5 ; 234,6$ & 2,9 & 1,000 \\
\hline Vitamina D $(\mu \mathrm{g})$ & $15,0^{\mathrm{e}}$ & 4,7 & 3,$5 ; 8,8$ & 100,0 & $15^{e}$ & 5,0 & 3,$3 ; 6,7$ & 100,0 & --- \\
\hline Vitamina E (mg) & $11,0^{e}$ & 13,7 & 11,$1 ; 17,8$ & 26,5 & $11^{\mathrm{e}}$ & 14,8 & 10,$9 ; 19,1$ & 35,3 & 0,549 \\
\hline Tiamina (mg) & $0,072^{\mathrm{ce,e,h}}$ & 1,9 & 1,$5 ; 2,3$ & 0,0 & $0,072^{\mathrm{c}, \mathrm{e}, \mathrm{h}}$ & 2,0 & 1,$6 ; 2,5$ & 0,0 & $-\ldots$ \\
\hline Riboflavina (mg) & $1,3^{\mathrm{c}}$ & 2,7 & 1,$8 ; 3,2$ & 11,8 & $1,5^{\circ}$ & 2,7 & 2,$2 ; 3,6$ & 8,8 & 1,000 \\
\hline Niacina (mg) & $1,3^{\mathrm{c}, \mathrm{e}} 8$ & 28,1 & 19,$6 ; 35,2$ & 0,0 & $1,3^{c, e, h}$ & 28,0 & 23,$3 ; 38,3$ & 0,0 & --- \\
\hline Piridoxina (mg) & $1,3^{\mathrm{c}}$ & 3,1 & 2,$4 ; 3,7$ & 0,0 & $1,5^{\circ}$ & 3,3 & 2,$4 ; 3,8$ & 5,9 & 0,500 \\
\hline Folato $(\mu \mathrm{g})$ & $320,0^{d}$ & 445,0 & 345,$9 ; 582,5$ & 14,7 & $520^{d}$ & 457,5 & 351,$6 ; 601,8$ & 58,8 & 0,001 \\
\hline Cobalamina $(\mu \mathrm{g})$ & $4,0^{\mathrm{e}}$ & 11,2 & 6,$7 ; 16,4$ & 2,9 & $4,5^{\mathrm{e}}$ & 9,5 & 7,$1 ; 12,1$ & 5,9 & 1,000 \\
\hline Cálcio (mg) & 860,$0 ; 750,0^{c, f, g}$ & 1132,3 & 840,$4 ; 1489,1$ & 20,6 & 860,$0 ; 750,0^{c, f, g}$ & 1355,6 & 909,$7 ; 1568,7$ & 11,8 & 0,453 \\
\hline Magnésio (mg) & $300,0^{e}$ & 420,0 & 333,$4 ; 482,5$ & 17,6 & $300,0^{e}$ & 457,8 & 370,$9 ; 582,5$ & 14,7 & 1,000 \\
\hline Ferro (mg) & $7,0^{\mathrm{c}}$ & 19,5 & 14,$9 ; 23,1$ & 0,0 & $7,0^{\circ}$ & 18,2 & 14,$2 ; 24,0$ & 0,0 &.- \\
\hline Zinco (mg) & $6,8^{d}$ & 14,6 & 11,$6 ; 16,5$ & 2,9 & $9,5^{d}$ & 16,2 & 12,$5 ; 18,2$ & 5,9 & 1,000 \\
\hline lodo (mg) & $150,0^{e}$ & 78,0 & 36,$4 ; 147,6$ & 76,5 & $200,0^{\mathrm{e}}$ & 115,6 & 83,$3 ; 167,2$ & 97,1 & 0,016 \\
\hline Cafeína (mg) & $400,0(37)$ & 84,2 & 42,$6 ; 115,5$ & 0,0 & $200,0(37)$ & 36,4 & 8,$4 ; 58,8$ & 0,0 & $-\ldots$ \\
\hline Fibra (g) & $25,0^{e}$ & 27,3 & 18,$1 ; 36,0$ & 44,1 & $28,0^{e}$ & 29,8 & 22,$2 ; 41,2$ & 47,1 & 1,000 \\
\hline $1 \mathrm{MJ}=238,83 \mathrm{kcal}$ & & & ${ }^{g} \geq 25$ anos & & & VET: & r energético total & & \\
\hline a AMDR: Acceptabl & Macronutrient Dis & tion Ranges & ${ }^{\mathrm{h}} \mathrm{mg} / \mathrm{MJ}$ & & & VR: V & de referência & & \\
\hline${ }^{\mathrm{b}} \mathrm{RI}$ : Reference Intal & e Ranges for Macr & rients & AGMI: Ácidos & Ġordos Monoinsatu & rados & Nota: & valores de $p$ cor & spondem à comp & ração das \\
\hline - AR: Average Requ & rements & & AGPI: Ácidos & Ardos Polinsatura & & perce & gens de inadequa & ão entre a pré-co & ceção e a \\
\hline${ }^{d}$ EAR: Estimated A & erage Requirement & & AGS: Ácidos & ordos Saturados & & gesta & & & \\
\hline e Al: Adequate Intak & & & HC: Hidratos & e Carbono & & & & & \\
\hline ' 24 anos & & & MJ: Megajou & & & & & & \\
\hline
\end{tabular}


o aumento de alguns valores de referência na gestação e a reduzida diferença no aporte nutricional entre o período pré-concecional e a gestação não se verificam muitas diferenças nas prevalências de inadequação nutricional. Apenas se identificaram diferenças com significado estatístico para o folato e o iodo, sendo a inadequação superior na gestação. Embora não apresente diferenças com significado estatístico, a mediana do aporte de cafeína reduziu para menos de metade na gravidez.

\section{DISCUSSÃO DOS RESULTADOS}

Neste estudo identificaram-se sete grávidas com aporte energético inferior às necessidades, ao contrário de Pinto et al. que não reportaram qualquer gestante com aporte energético insuficiente (8).

Identificamos também diversos nutrientes com elevada proporção de inadequação, o que para além de resultar dos hábitos alimentares desta população, deve ser interpretado em termos comparativos tendo em consideração a escolha de diferentes valores de referência em diferentes estudos. Entre a gestação e a pré-conceção apenas se verificam diferenças com significado estatístico na adequação nutricional dos folatos e iodo, sendo a percentagem de inadequação por defeito superior na gravidez. Este resultado é expectável porque, em conjunto com a vitamina $A$, estes são os micronutrientes cujas necessidades mais aumentam na gravidez, o aumento do folato e iodo é de $200 \mu \mathrm{g}$ e $50 \mathrm{mg}$, respetivamente, embora se tenha verificado que o seu aporte nos dois momentos avaliados era semelhante. É habitual a suplementação de ácido fólico e de iodo antes da conceção e durante a gestação, pelo que se destaca a importância da suplementação, particularmente para melhorar a adequação nutricional ("Perfil de suplementação antes e durante a gestação: estudo de acompanhamento na ilha do Faial") (33).

Relativamente aos hidratos de carbono, verificou-se que mais de metade da amostra, nos dois momentos de avaliação, apresentava um aporte inadequado, por defeito, sendo superior no período pré-gestacional. É de salientar que os resultados de outros estudos revelam uma proporção de inadequação menor antes da conceção e durante a gravidez $(7,8,16,26,31)$.

Os lípidos foram o macronutriente com maior proporção de inadequação, por excesso, especialmente na gravidez. Antes da conceção a proporção de inadequação $(73,5 \%)$ foi bastante superior comparativamente a outros estudos (8, 30). São vários os estudos que demonstram que durante a gravidez há uma elevada prevalência de aporte excessivo de gordura, contudo apresentam valores de inadequação mais baixos $(8,14,15)$. Não verificamos alterações relevantes na contribuição percentual do valor energético total proveniente dos diferentes tipos de ácidos gordos. Na gestação, a percentagem relativa aos ácidos gordos polinsaturados manteve-se $(6,1 \%)$, mas houve um aumento de 0,7 e 1,0 pontos percentuais nos ácidos gordos saturados (de 10,1 para 10,8\%) e monoinsaturados (de 17,5 para $18,5 \%$ ), respetivamente.

Relativamente à vitamina $\mathrm{D}$, nenhuma das participantes teve um aporte adequado antes ou durante a gestação. Os resultados de outros estudos demonstram que na gravidez a prevalência de inadequação desta vitamina tende a ser bastante elevada $(14,15,21)$. É de salientar que o nível sérico da vitamina D não corresponde apenas ao seu aporte, pelo que os níveis de inadequação podem ser inferiores, especialmente no verão, dado que nesta estação a exposição solar poderá ser a principal fonte desta vitamina (29), pelo que a interpretação destes valores de inadequação deverá ser feita com cautela.

Neste estudo verificou-se um baixo aporte de folatos antes e durante a gravidez, o que reforça a importância da sua suplementação. No estudo de Pinto et al., a prevalência de inadequação desta vitamina foi elevada nos dois momentos, mas superior na gestação $(90,8 \%)$ (17). Looman et al. também encontraram uma elevada percentagem de défice no período pré-gestacional (80,7\%) (34).

Relativamente ao iodo foi possível identificar um aporte inadequado antes e durante a gestação. Importa referir que o QFA não é o melhor instrumento para analisar o aporte deste mineral, não só porque não tem em conta a utilização de sal iodado, mas também porque contém informação nutricional proveniente de tabelas de composição de alimentos não nacionais. No estudo de Rodriguez-Bernal et al. 24,3\% das grávidas apresentou inadequação deste mineral (21).

Quanto à fibra, nos dois momentos de análise foi possível verificar a existência de inadequação, por defeito, sendo ligeiramente superior $(47,1 \%)$ na gravidez. Comparativamente com a mediana do IAN-AF, o valor deste estudo na pré-conceção é superior (30). Segundo AbuSaad et al. foi encontrada uma prevalência de inadequação superior na gestação, mas Dubois et al. não detetaram qualquer inadequação deste nutriente neste mesmo período $(14,35)$.

Tal como todos os trabalhos de investigação, o presente também apresenta limitações, das quais se destaca o facto de ter sido foi realizado em mulheres com um elevado nível de literacia e que residem apenas na ilha do Faial, o que condiciona a extrapolação dos resultados. Considerando o desenho do estudo houve a necessidade de excluir algumas inquiridas, o que poderá ter influenciado os resultados relativos ao consumo alimentar e respetivo aporte nutricional. Ao longo da aplicação dos dois QFA, as inquiridas foram informadas várias vezes sobre o período a que estava a ser aplicado esta ferramenta, contudo os dados foram recolhidos de forma retrospetiva o que poderá comprometer a acuidade da informação recolhida. Para além disso, está descrito que o uso destes questionários tende a sobrestimar a ingestão alimentar (36), pelo que há a possibilidade da inadequação nutricional por défice estar subestimada e da inadequação nutricional por excesso ser na realidade inferior ao que é descrito nos resultados. $\mathrm{Na}$ avaliação do consumo alimentar e do aporte nutricional antes e durante a gestação foi estudada a mesma amostra, o que minimizou o viés associado às características das participantes.

\section{CONCLUSÕES}

Neste estudo foi possível constatar que as mulheres que residem no Faial apresentam uma elevada inadequação nutricional quer em macro quer em micronutrientes. Os nutrientes com maior inadequação antes da conceção foram: vitamina D, iodo, lípidos, hidratos de carbono e fibra. Todos estes micronutrientes tiveram uma inadequação por defeito, à exceção dos lípidos que apresentaram inadequação por excesso. Na gestação, para além dos nutrientes mencionados anteriormente, os folatos também apresentaram uma elevada percentagem de inadequação por defeito.

Os resultados deste estudo reforçam a importância da adesão à suplementação, nomeadamente de iodo e ácido fólico que por sua vez são de distribuição gratuita na região, fomenta a necessidade da sensibilização para a intervenção nutricional no âmbito da Saúde Materna e Planeamento Familiar, de forma a atuar com a conveniente precocidade.

\section{REFERÊNCIAS BIBLIOGRÁFICAS}

1. Langley-Evans SC. Nutrition in early life and the programming of adult disease: a review. J Hum Nutr Diet. 2015;28 Suppl 1:1-14.

2. Nutrition Working G, O'Connor DL, Blake J, Bell R, Bowen A, Callum J, et al. Canadian Consensus on Female Nutrition: Adolescence, Reproduction, Menopause, and Beyond. J Obstet Gynaecol Can. 2016;38(6):508-54 e18. 
3. Stephenson J, Patel D, Barrett G, Howden B, Copas A, Ojukwu O, et al. How do women prepare for pregnancy? Preconception experiences of women attending antenatal services and views of health professionals. PLoS One. 2014;9(7):e103085. 4. Agosti M, Tandoi F, Morlacchi L, Bossi A. Nutritional and metabolic programming during the first thousand days of life. Pediatr Med Chir. 2017;39(2):157.

5. Teixeira D, Marinho R, Mota I, Castela I, Morais J, Pestana D, et al. Alimentação e Nutrição na Gravidez 2021. Available from: https://nutrimento.pt/activeapp/wp-content/ uploads/2021/03/ManualGravidez_Final-3Marc\%CC\%A702021.pdf.

6. Harika R, Faber M, Samuel F, Kimiywe J, Mulugeta A, Eilander A. Micronutrient Status and Dietary Intake of Iron, Vitamin A, lodine, Folate and Zinc in Women of Reproductive Age and Pregnant Women in Ethiopia, Kenya, Nigeria and South Africa: A Systematic Review of Data from 2005 to 2015. Nutrients. 2017;9(10).

7. Lundqvist A, Johansson I, Wennberg A, Hultdin J, Hogberg U, Hamberg K, et al. Reported dietary intake in early pregnant compared to non-pregnant women - a crosssectional study. BMC Pregnancy Childbirth. 2014;14:373.

8. Pinto $\mathrm{E}$, Barros $\mathrm{H}$, dos Santos Silva I. Dietary intake and nutritional adequacy prior to conception and during pregnancy: a follow-up study in the north of Portugal. Public Health Nutr. 2009;12(7):922-31.

9. Dean SV, Lassi ZS, Imam AM, Bhutta ZA. Preconception care: nutritional risks and interventions. Reprod Health. 2014;11 Suppl 3:S3.

10. Grieger JA, Grzeskowiak LE, Clifton VL. Preconception dietary patterns in human pregnancies are associated with preterm delivery. J Nutr. 2014;144(7):1075-80.

11. Stephenson J, Heslehurst N, Hall J, Schoenaker D, Hutchinson J, Cade JE, et al. Before the beginning: nutrition and lifestyle in the preconception period and its importance for future health. Lancet. 2018;391(10132):1830-41.

12. Blumfield ML, Hure AJ, Macdonald-Wicks L, Smith R, Collins CE. Systematic review and meta-analysis of energy and macronutrient intakes during pregnancy in developed countries. Nutr Rev. 2012;70(6):322-36.

13. Blumfield ML, Hure AJ, Macdonald-Wicks L, Smith R, Collins CE. A systematic review and meta-analysis of micronutrient intakes during pregnancy in developed countries. Nutr Rev. 2013;71(2):118-32.

14. Dubois L, Diasparra M, Bedard B, Colapinto CK, Fontaine-Bisson B, Morisset AS, et al. Adequacy of nutritional intake from food and supplements in a cohort of pregnant women in Quebec, Canada: the 3D Cohort Study (Design, Develop, Discover). Am J Clin Nutr. 2017;106(2):541-8.

15. Dubois L, Diasparra M, Bedard B, Colapinto CK, Fontaine-Bisson B, Tremblay RE, et al. Adequacy of nutritional intake during pregnancy in relation to prepregnancy BMI: results from the 3D Cohort Study. Br J Nutr. 2018;120(3):335-44.

16. Gernand AD, Schulze KJ, Stewart CP, West KP, Jr., Christian P. Micronutrient deficiencies in pregnancy worldwide: health effects and prevention. Nat Rev Endocrinol. 2016;12(5):274-89.

17. Goletzke J, Buyken AE, Louie JC, Moses RG, Brand-Miller JC. Dietary micronutrient intake during pregnancy is a function of carbohydrate quality. Am $\mathrm{J}$ Clin Nutr. 2015;102(3):626-32.

18. Gomes CB, Malta MB, Corrente JE, Benicio MH, Carvalhaes MA. [High prevalence of inadequate calcium and vitamin $\mathrm{D}$ dietary intake in two cohorts of pregnant women]. Cad Saude Publica. 2016;32(12):e00127815.

19. Groth SW, Stewart PA, Ossip DJ, Block RC, Wixom N, Fernandez ID. Micronutrient Intake Is Inadequate for a Sample of Pregnant African-American Women. J Acad Nutr Diet. 2017;117(4):589-98.

20. Hatzopoulou K, Filis V, Grammatikopoulou MG, Kotzamanidis C, Tsigga M. Greek pregnant women demonstrate inadequate micronutrient intake despite supplement use. J Diet Suppl. 2014;11(2):155-65.

21. Rodriguez-Bernal CL, Ramon R, Quiles J, Murcia M, Navarrete-Munoz EM, Vioque $J$, et al. Dietary intake in pregnant women in a Spanish Mediterranean area: as good as it is supposed to be? Public Health Nutr. 2013;16(8):1379-89.

22. Bath SC, Steer CD, Golding J, Emmett P, Rayman MP. Effect of inadequate iodine status in UK pregnant women on cognitive outcomes in their children: results from the Avon Longitudinal Study of Parents and Children (ALSPAC). Lancet. 2013;382(9889):331-7.
23. Blumfield ML, Hure AJ, MacDonald-Wicks LK, Smith R, Simpson SJ, Giles WB, et al. Dietary balance during pregnancy is associated with fetal adiposity and fat distribution. Am J Clin Nutr. 2012;96(5):1032-41.

24. Christian P, Stewart CP. Maternal micronutrient deficiency, fetal development, and the risk of chronic disease. J Nutr. 2010;140(3):437-45.

25. Gernand AD, Christian P, Schulze KJ, Shaikh S, Labrique AB, Shamim AA, et al. Maternal nutritional status in early pregnancy is associated with body water and plasma volume changes in a pregnancy cohort in rural Bangladesh. J Nutr. 2012;142(6):1109-15. 26. Lopes C. Reprodutibilidade e Validação de um questionário semi-quantitativo de frequência alimentar. In: Alimentação e enfarte agudo do miocárdio: um estudo caso-controlo de base populacional. Tese de Doutoramento. Universidade do Porto 2000. p.79-115.27.

27. Lopes C, Aro A, Azevedo A, Ramos E, Barros H. Intake and adipose tissue composition of fatty acids and risk of myocardial infarction in a male Portuguese community sample. J Am Diet Assoc. 2007;107(2):276-86.

28. Pinto E, Severo M, Correia S, dos Santos Silva I, Lopes C, Barros H. Validity and reproducibility of a semi-quantitative food frequency questionnaire for use among Portuguese pregnant women. Matern Child Nutr. 2010;6(2):105-19.

29. EFSA (European Food Safety Authority), 2017. Dietary Reference Values for nutrients. Summary Report. EFSA supporting publication 2017:e15121. 98 pp. doi:10.2903/sp.efsa.2017.e15121.

30. Lopes C ea, Inquérito Alimentar Nacional e de Atividade Física IAN-AF 2015-2016. Relatório de resultados. 2017, Universidade do Porto: Porto.

31. Trumbo P, Schlicker S, Yates AA, Poos M, Food, Nutrition Board of the Institute of Medicine TNA. Dietary reference intakes for energy, carbohydrate, fiber, fat, fatty acids, cholesterol, protein and amino acids. J Am Diet Assoc. 2002;102(11):1621-30. 32. Trumbo P, Schlicker S, Yates AA, Poos M, Food and Nutrition Board of the Institute of Medicine TNA. Dietary reference intakes for energy, carbohydrate, fiber, fat, fatty acids, cholesterol, protein and amino acids. J Am Diet Assoc. 2002;102(11):1621-30. 33. Viveiros F, Poinhos R, Afonso C. Perfil de suplementação antes e durante a gestação: estudo de acompanhamento na ilha do Faial. Acta Portuguesa de Nutrição 2021, 26, 06-09. 2021.

34. Looman M, Schoenaker D, Soedamah-Muthu SS, Mishra GD, Geelen A, Feskens EJM. Pre-pregnancy dietary micronutrient adequacy is associated with lower risk of developing gestational diabetes in Australian women. Nutr Res. 2019;62:32-40.

35. Abu-Saad K, Shahar DR, Fraser D, Vardi H, Friger M, Bolotin A, et al. Adequacy of usual dietary intake and nutritional status among pregnant women in the context of nutrition transition: the DEPOSIT Study. Br J Nutr. 2012;108(10):1874-83.

36. Cade J, Thompson R, Burley V, Warm D. Development, validation and utilisation of food-frequency questionnaires - a review. Public Health Nutr. 2002;5(4):567-87. 37. EFSA NDA Panel (EFSA Panel on Dietetic Products NaA, 2015. Scientific Opinion on the safety of caffeine. EFSA Journal 2015;13(5):4102, 120 pp. doi:10.2903/j. efsa.2015.4102. 